\title{
EXTENSIVE SUBGLOTTIC STENOSIS IN A YOUNG PATIENT DIAGNOSED WITH GRANULOMATOSIS WITH POLYANGIITIS - CASE REPORT
}

\author{
André Busato da Costa ${ }^{1, \star}$, Millena Almeida Souza Ramos ${ }^{1}$, Bruna Burko Rocha Chu $^{1}$ \\ 1.Universidade Positivo, Curitiba (SP), Brazil. \\ *Corresponding author: andre-busato@hotmail.com
}

\section{BACKGROUND}

Granulomatosis with polyangiitis (GPA) is a systemic granulomatous vasculitis that affects the upper and lower respiratory tracts and the kidneys. The disease is associated with antineutrophil cytoplasmatic antibody (ANCA). Most individuals are between 45 and 65 years old at the diagnosis and the prevalence varies according to population studied, which may be as rare as 2.3 cases per million inhabitants. Subglottic stenosis is a manifestation of tracheobronchial GPA and may be severe enough to necessitate tracheostomy. We present a case of a young male with extensive subglottic stenosis.

\section{CASE REPORT}

A 22-year-old male started with hoarseness followed by dyspnea four years ago. He tried several treatments with antibiotics and intranasal corticosteroids with no response. Later, he developed tinnitus, knee and distal interphalangeal pain, and the dyspnea got worse. The patient denied comorbidities, smoking and previous invasive mechanic ventilation. He reported several episodes of conjunctivitis and otitis media during childhood. The laboratory tests showed normal inflammatory markers, creatinine, antinuclear antibody and rheumatoid factor; c-ANCA and p-ANCA antibodies had inconclusive results. The bronchoscopy revealed subglottic stenosis (Figure 1) obstructing more than $50 \%$ of the tracheal lumen. The computed tomography (CT) finding were multiple pulmonary cysts, ground glass opacities and subglottic stenosis (Figures 2 and 3). Tracheal biopsy revealed atypic lymphoproliferative process. Immunohistochemistry findings were chronic lymphohistiocytic inflammatory process with fibrosis.

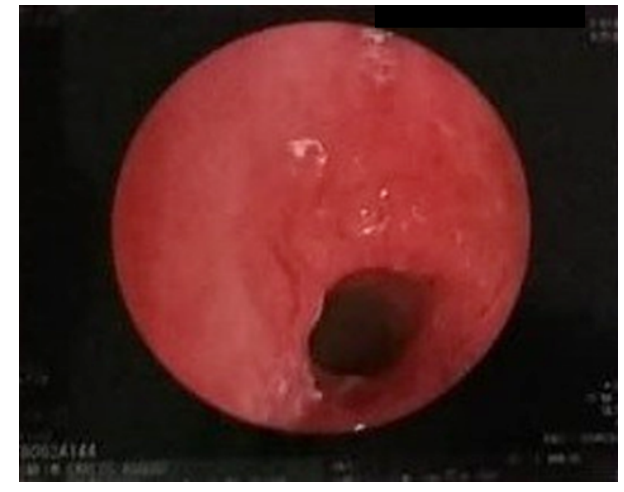

Figure 1. Bronchoscopy revealing subglottic stenosis.

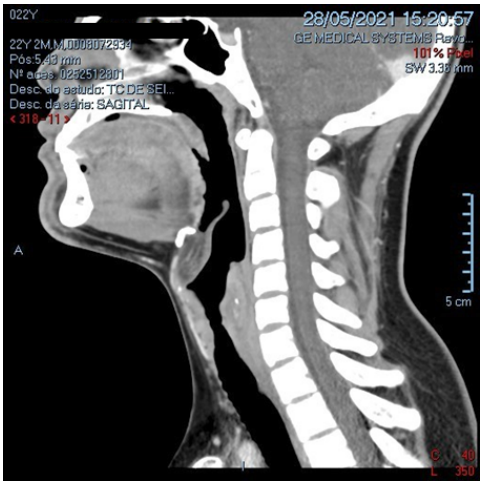

Figure 2. Cervical CT on sagittal plane exhibiting subglottic stenosis.

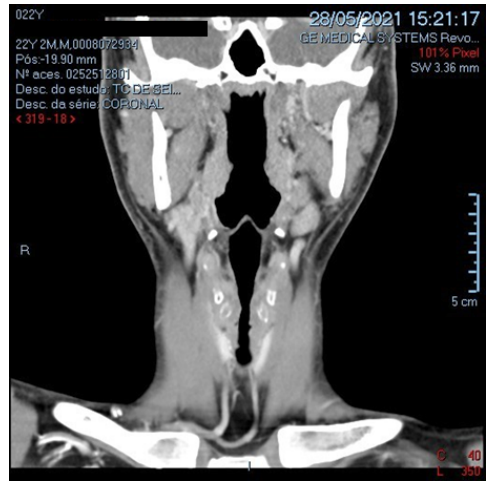

Figure 3. Cervical CT on axial plane exhibiting subglottic stenosis.

\section{CONCLUSION}

Granulomatosis with polyangiitis can involve almost any organ or tissue, but the respiratory tract is the most commonly affected. The patient can present with nonspecific symptoms, including hoarseness and dyspnea. Thus, the disease can be misdiagnosed initially as an infection. Subglottic stenosis may be severe enough to necessitate tracheostomy. That is why the occurrence of recurrent otorhinolaryngological symptoms should raise the hypothesis of GPA for early treatment and to avoid serious morbidities. ANCA should be performed in any individual with symptoms suggestive of a vasculitis, but its absence does not exclude the diagnosis. Whenever possible, the diagnosis should be confirmed by biopsy.

\section{KEYWORDS}

Granulomatosis with polyangiitis, Subglottic stenosis. 